See discussions, stats, and author profiles for this publication at: https://www.researchgate.net/publication/320885790

\title{
Introducing ADELE: a personalized intelligent companion
}

Conference Paper · November 2017

DOI: $10.1145 / 3139491.3139492$

CITATIONS

0

7 authors, including:

Emer Gilmartin

Trinity College Dublin

37 PUBLICATIONS 142 CITATIONS

SEE PROFILE

8. Vincent P Wade

Trinity College Dublin

282 PUBLICATIONS 2,571 CITATIONS

SEE PROFILE

Some of the authors of this publication are also working on these related projects:

Project $\quad$ ADAPT Centre for Digital Content Technology Research View project

Project MediLink View project
READS

75

Séamus Lawless

Trinity College Dublin

86 PUBLICATIONS 602 CITATIONS

SEE PROFILE 


\section{Introducing ADELE: A Personalized Intelligent Companion}

\author{
Brendan Spillane \\ ADAPT Centre, Trinity College \\ Dublin, Ireland \\ brendan.spillane@adaptcentre.ie \\ Ketong $\mathrm{Su}$ \\ ADAPT Centre, Trinity College \\ Dublin, Ireland \\ kesu@tcd.ie
}

\author{
Emer Gilmartin \\ ADAPT Centre, Trinity College \\ Dublin, Ireland \\ gilmare@tcd.ie \\ Benjamin R. Cowan \\ ADAPT Centre, University College \\ Dublin, Ireland \\ benjamin.cowan@ucd.ie \\ Vincent Wade \\ ADAPT Centre, Trinity College \\ Dublin, Ireland \\ vincent.wade@adaptcentre.ie
}

\author{
Christian Saam \\ ADAPT Centre, Trinity College \\ Dublin, Ireland \\ christian.saam@adaptcentre.ie \\ Séamus Lawless \\ ADAPT Centre, Trinity College \\ Dublin, Ireland \\ seamus.lawless@adaptcentre.ie
}

\begin{abstract}
This paper introduces ADELE, a Personalized Intelligent Companion designed to engage with users through spoken dialog to help them explore topics of interest. The system will maintain a user model of information consumption habits and preferences in order to (1) personalize the user's experience for ongoing interactions, and (2) build the user-machine relationship to model that of a friendly companion. The paper details the overall research goal, existing progress, the current focus, and the long term plan for the project.
\end{abstract}

\section{CCS CONCEPTS}

- Human-centered computing $\rightarrow$ Human computer interaction (HCI); User models; • Information systems $\rightarrow$ Personalization;

\section{KEYWORDS}

Intelligent Companion, Personalization, Spoken Dialog System

\section{ACM Reference Format:}

Brendan Spillane, Emer Gilmartin, Christian Saam, Ketong Su, Benjamin R. Cowan, Séamus Lawless, and Vincent Wade. 2017. Introducing ADELE: A Personalized Intelligent Companion. In Proceedings of 1st ACM SIGCHI International Workshop on Investigating Social Interactions with Artificial Agents (ISIAA'17). ACM, New York, NY, USA, 2 pages. https://doi.org/10. $1145 / 3139491.3139492$

\section{INTRODUCTION}

The ADELE Personalized Intelligent Companion is a virtual agent which will be capable of engaged, yet natural and informed, casual conversation. It will assist a user not only in small tasks that the user initiates but in more prolonged dialogue to inform the user of news,

Permission to make digital or hard copies of part or all of this work for personal or classroom use is granted without fee provided that copies are not made or distributed for profit or commercial advantage and that copies bear this notice and the full citation on the first page. Copyrights for third-party components of this work must be honored.

For all other uses, contact the owner/author(s).

ISIAA'17, November 13, 2017, Glasgow, UK

(C) 2017 Copyright held by the owner/author(s)

ACM ISBN 978-1-4503-5558-2/17/11 \$\$15.00

https://doi.org/10.1145/3139491.3139492 events and other information of interest through conversation, using a mix of user and agent-initiated interaction.

To achieve these aims the companion will engage the user in conversations that employ a natural mix of linguistic and paralinguistic devices to give and seek information but also to entertain. These may carry elements of task execution but will to a large extent comprise social talk. Accordingly, topic, style and register must be varied at levels ranging from lexical and syntactic to socioand dialectal pronunciation, to tone of voice, speed and rhythm.

ADELE is intended to converse with the user on topics relating to their personal interests, current events and social feeds, while in turn learning about the user. With repeated interaction, the system will build a profile of the user's interests and also how the user wants to get information in order to provide editorialized summaries of news articles personalized to the user's consumption preferences in terms of source, granularity, depth, and detail. To achieve these aims ADELE will model:

(1) user preferences - topics, sources, and register of delivery

(2) user characteristics - biographic information etc.

(3) current context - user cognitive and affective state etc.

Currently there is commercial focus on intelligent personal assistants with the advent of Apple's Siri, Google Assistant, Amazon's Alexa and Microsoft's Cortana. However, these AI personal assistants are focused on helping users to complete specific tasks with a defined start and end. Examples include ordering a product online or scheduling an appointment. ADELE differentiates itself by focusing on tasks which keep the user abreast of current events or instigate conversations on hot topics in their social networks.

\section{RESEARCH GOAL}

The research goal is to discover if it is possible through a stochastic spoken dialog system based on Artificial Neural Networks (ANN) and using personalization to create a digital companion which is capable of communicating through informal yet informed social dialog on a variety of topics of interest to a user over a prolonged timescale. Evaluating ADELE as a companion for the elderly is a likely research focus. 


\section{CURRENT PROGRESS}

The project has focused on (1) The collection of a initial dataset with social and task dimensions necessary to begin training an ANN in the intricacies of social dialog and to inform the team of the requirements of the dataset necessary to train an ANN capable of complex and sustained spoken dialog. (2) The design and development of the ANN dialog system.

A preliminary corpus of text dialogs was collected over two months in mid 2016. Naive participants were paired up remotely using a text based interface to perform an information gap role play activity modelling a first encounter. Each participant was assigned a fictitious persona and required to converse with another participant in order to discover biographical information. Participants were awarded points for each utterance, for recording correct details about the other participant's adopted persona and for discovering details in common. The dialog interface was a Google Chrome extension for the team collaboration tool Slack ${ }^{1}$. In total 389 conversations were included in the final dataset.

The conversations were annotated for dialog acts using the ISO 24617-2 standard [2]. During this process it was discovered that the ISO standard was inefficient for annotating the complex greeting and leave-taking sequences in the collected conversations. Although most dialog annotation schemes are modelled on task-based dialog, they usually include some tags for social interaction; as in the Social Obligation Management (SOM) functions in the ISO standard. However, tag sets for social dialog acts are limited to formalities such as greetings, apologies and farewells. As ADELE needs to be capable of instigating and sustaining prolonged social dialog, there is a need for adequate SOM coverage of formalities, and also for consideration of talk which does not have a clear instrumental goal. As a first step, new acts for greeting and leave-taking sequences in the corpus were devised [3]

\section{BUILDING AND TRAINING THE SYSTEM}

The dialog system comprises the following modules: Natural Language Understanding (NLU), State Tracking (ST), Policy (P), Natural Language Generation (NLG) and Database Query (DBQ).

The modules for NLU, P, NLG and ST are currently RNN Sequence to Sequence (S2S) transduction models with attention [1] The modules communicate over semantics vectors with a shared vocabulary. Instead of designing custom NNs for different modules the problems of state tracking and policy learning were cast in a way that makes them amenable to sequence to sequence learning. The ST keeps track of the slots that are used to constrain the query into the database, the Policy module decides what output is to be generated by the NLG depending on the dialog history and the result of the DBQ. NLU and NLG translate from natural language to internal semantics and vice versa.

The internal semantics of the current system is based on CUED Standard Dialog Acts [6]. This means that for the sequence to sequence learning, a corpus with explicit annotation of these acts is required. The specific format of these annotations is however not important. The first version is trained on data from the 2nd and 3rd Dialog State Tracking Challenge [4][5]. The internal semantics

\footnotetext{
${ }^{1}$ https://slack.com/
}

allow the modules to be trained separately and are easily inspected, however they present a challenge for end-to-end training.

While the system is so far text based it was trained on transcripts of dialogs equivalent to optimal one-best hypotheses from Automatic Speech Recognition (ASR). Future versions will be able to receive alternative recognition hypotheses from ASR in the form of n-best lists or word lattices as input.

\section{EVALUATION}

Currently our focus is on the evaluation of the first iteration of the ADELE Personalized Intelligent Companion.

In the evaluation scenario, ADELE will represent one of several virtual characters with specific traits based on those from the previous experiment. The participant will also impersonate a given persona with specific traits. A text based conversation will be set up between ADELE and each participant using a web interface for the evaluation. ADELE and the participant will each be an interlocutor in a one-on-one conversation. ADELE will inform the opposing interlocutor about its own characteristics while at the same time trying to elicit the given character traits of the participant's persona.

\section{FUTURE}

The next iteration of ADELE will add speech to the dialog system. A larger training corpus, based on the requirements from this evaluation, will also be added to the training dataset. A user model for personalization will also be added to the system. The success of implicitly and explicitly adding user information to this will be measured in the next evaluation.

\section{CONCLUSIONS}

The ADELE project is an ongoing collaboration between computational linguistics, HCI researchers, and computer scientists specializing in personalization. The first iteration of the ADELE dialog system is undergoing evaluation with a view to forming requirements for an expanded training dataset and the next iteration.

\section{ACKNOWLEDGMENTS}

This research is supported by the Science Foundation Ireland (Grant $13 / \mathrm{RC} / 2106$ ) and the ADAPT Centre (www.adaptcentre.ie) at Trinity College, Dublin.

\section{REFERENCES}

[1] Dzmitry Bahdanau, Kyunghyun Cho, and Yoshua Bengio. 2014. Neural Machine Translation by Jointly Learning to Align and Translate. arXiv:1409.0473 [cs, stat] (Sept. 2014). http://arxiv.org/abs/1409.0473 00570 arXiv: 1409.0473.

[2] ISO DIS. 2010. 24617-2 (2010) Language resource management-Semantic annotation framework (SemAF), Part 2: Dialogue acts. ISO, Geneva, fanuary (2010).

[3] E. Gilmartin, B. Spillane, M. O’Reilly, Saam C., K. Su, K. Levacher, L. Cerrato, B R. Cowan, L M. Clark, A. Calvo, N. Campbell, and V Wade. 2017. Annotation of Greeting and Leave-taking in Social Text Dialogues Using ISO 24617-2. In Thirteenth foint ACL - ISO Workshop on Interoperable Semantic Annotation. Montpellier, France.

[4] M. Henderson, B. Thomson, and J. D. Williams. 2014. The Second Dialog State Tracking Challenge. Association for Computational Linguistics, Philadelphia, PA, U.S.A. http://www.aclweb.org/anthology/W14-4337

[5] M. Henderson, B. Thomson, and J. D. Williams. 2014. The third Dialog State Tracking Challenge. In 2014 IEEE Spoken Language Technology Workshop (SLT). 324-329. https://doi.org/10.1109/SLT.2014.7078595

[6] Steve Young. 2007. CUED Standard Dialogue Acts. Technical Report. Cambridge University Engineering Department. 